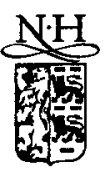

ELSEVIER

Nuclear Physics B 559 (1999) 3-16

\title{
Top-quark physics in six-quark final states at the Next Linear Collider
}

\author{
F. Gangemi ${ }^{a, b}$, G. Montagna ${ }^{a, b}$, M. Moretti ${ }^{c, d}$, O. Nicrosini ${ }^{\text {b,a }}$, \\ F. Piccinini ${ }^{b, a}$ \\ a Dipartimento di Fisica Nucleare e Teorica, Università di Pavia, via A. Bassi 6, Pavia, Italy \\ ${ }^{b}$ Istituto Nazionale di Fisica Nucleare, Sezione di Pavia, via A. Bassi 6, Pavia, Italy \\ c CERN - Theory Division, Geneva, Switzerland \\ d Dipartimento di Fisica, Università di Ferrara and INFN, Sezione di Ferrara, Ferrara, Italy
}

Received 11 May 1999; accepted 30 July 1999

\begin{abstract}
The processes of six-quark production with one $b \bar{b}$ pair are studied by means of a complete tree-level electroweak calculation. The top-quark signal is examined: the importance of electroweak backgrounds, of the order of $10 \%$ above the $t \bar{t}$ threshold and of about $30 \%$ of the purely electroweak signal at threshold, is further stressed by studying the dependence of the cross section at threshold on the Higgs mass in the range between $100 \mathrm{GeV}$ and $185 \mathrm{GeV}$, and finding variations of the order of $10 \%$. In the study of some event-shape variables, a strong effect of initial-state radiation is found, in particular for the thrust distribution, which is studied for several centre-of-mass energies at the TeV scale. The effectiveness of cuts on the thrust for isolating QCD backgrounds, as pointed out by some authors, is confirmed also in the presence of electroweak backgrounds and initial-state radiation. (C) 1999 Elsevier Science B.V. All rights reserved.
\end{abstract}

PACS: $02.70 . \mathrm{Lq} ; 13.85 . \mathrm{Hd} ; 14.80 . \mathrm{Bn}$

Keywords: Electron-positron collisions; Six fermions; Top-quark; Higgs boson; Monte Carlo

\section{Introduction}

Many important signals to be studied at future high energy colliders, NLC [1] and LHC [2], will have a large number of particles in the final state. In particular, the processes with six fermions in the final state will be of great importance for several tests of the Standard Model, such as the studies of top-quark and electroweak gauge bosons, as well as the search for an intermediate-mass Higgs boson. Such processes are already of great interest at the Tevatron collider [3] in connection with top-quark 
Table 1

Six-quark final states with one $b \bar{b}$ pair. The notations CC (charged currents) and NC (neutral currents) refer to the currents formed by the quark flavours other than $b$

\begin{tabular}{lll}
\hline CC only & CC and NC & NC only \\
\hline$b \bar{b} u \bar{d} \bar{c} s$ & $b \bar{b} u \bar{d} \bar{u} d$ & $b \bar{b} u \bar{u} s \bar{s}, b \bar{b} c \bar{c} d \bar{d}$ \\
$b \bar{b} \bar{u} d c \bar{s}$ & $b \bar{b} c \bar{s} \bar{c} s$ & $b \bar{b} u \bar{u} u \bar{u}, b \bar{b} c \bar{c} c \bar{c}$ \\
& & $b \bar{b} d \bar{d} d \bar{d}, b \bar{b} s \bar{s} s \bar{s}$ \\
& $b \bar{b} u \bar{u} c \bar{c}$ \\
& $b \bar{b} d \bar{d} s \bar{s}$ \\
\hline
\end{tabular}

physics. Theoretical studies of six-fermion $(6 f)$ processes by means of complete treelevel calculations have only very recently appeared in the literature [4-9], where topquark physics, Higgs physics and $W W Z$ production have been addressed.

All these studies clearly demonstrate that complete calculations are important for a precise determination of the cross sections, and for the development of reliable event generators, whenever accurate evaluations of interference, off-shellness and background effects, as well as spin correlations, are important.

In view of the precision measurements of the top-quark properties we have to analyse, among the $6 f$ signatures, the ones containing a $b \bar{b}$ pair and two charged currents, as the top-quark decays almost exclusively into a $W$ boson and a $b$ quark. Semi leptonic signatures have already been considered in Refs. $[4,5,10]$. It is then of great interest to carefully evaluate the size of the totally hadronic, six-quark $(6 q)$ contributions to integrated cross sections and distributions as well as to determine their phenomenological features. The aim of the present study is to make a first quantitative analysis in this context, for what concerns the full set of electroweak contributions to a class of $e^{+} e^{-}$ annihilation processes related to top-quark physics. Special emphasis will be given to the determination and to the analysis of the topology of the events considered, so as to characterize them, as far as possible, against the QCD backgrounds.

Looking at an experimental situation where the $b$-tagging technique can be applied, it is meaningful to distinguish the $6 q$ final states containing one $b \bar{b}$ pair, of the form $b \bar{b} q \bar{q}^{\prime} q^{\prime \prime} \bar{q}^{\prime \prime \prime}$, from those containing two or three $b \bar{b}$ pairs, respectively of the form $b \bar{b} b \bar{b} q \bar{q}$ and $b \bar{b} b \bar{b} b \bar{b}$. The last two kinds of processes are not relevant to top-quark production, as they contain no charged currents.

In the present study the signatures with one $b \bar{b}$ pair are considered and the full set of purely electroweak contributions is taken into account. These processes can be further divided into three subsets (although in realistic predictions they cannot be treated separately), which are shown in Table 1: concerning the quark flavours other than $b$, only charged currents are involved in the first subset, both charged and neutral currents in the second one, and only neutral currents in the third one. The total number of tree-level Feynman diagrams involved in the complete electroweak calculation amounts to several hundreds. Such a complexity is unavoidable, as will be shown, if an accuracy of $1 \%$ is to be reached. The diagrams with top-quark production, which will be referred to as signal diagrams, are shown in Fig. 1. They con- 


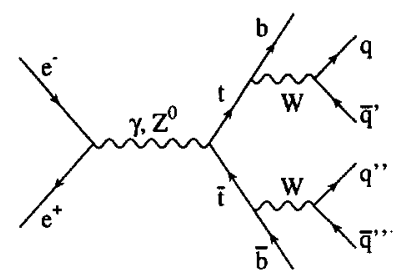

Fig. 1. The two Feynman diagrams with $t \bar{t}$ production.

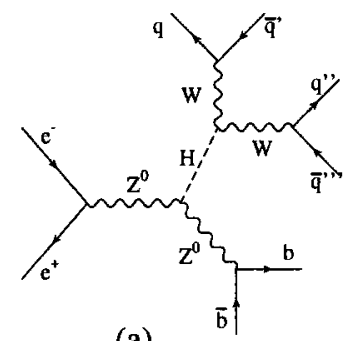

(a)

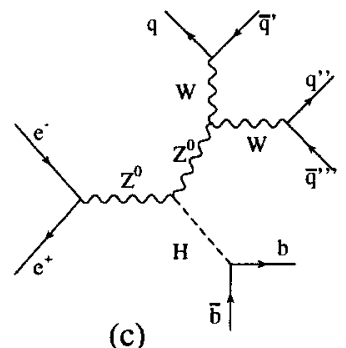

(c)

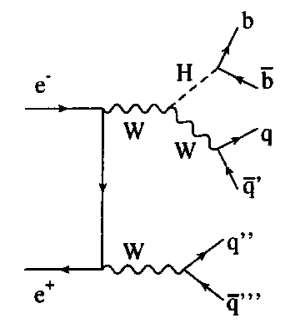

(d)

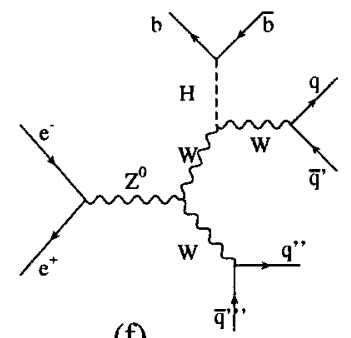

(f)

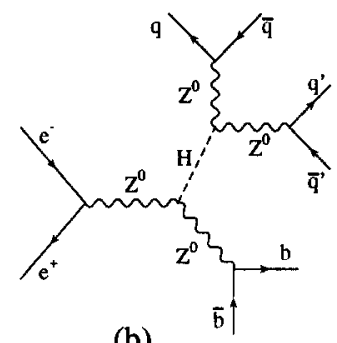

(b)

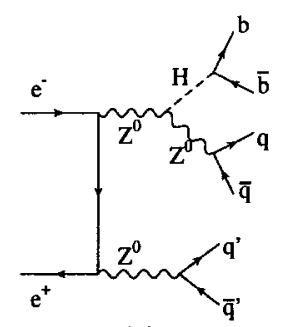

(e)

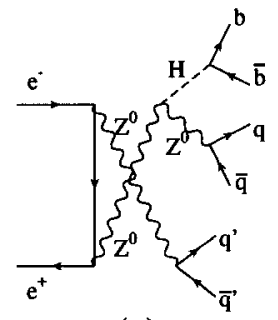

(g)

Fig. 2. Leading Higgs contributions, divided into charged-current terms, on the left, and neutral-current terms, on the right (where charged and neutral currents are referred to quark flavours other than $b$ ). Other diagrams, obtained by attaching the Higgs line to the other possible gauge-boson line in the diagrams (d), (e), (f), and (g), are understood.

tribute to the processes in the first two columns of Table 1, but not to those in the third.

All the processes receive contributions from diagrams of Higgs production, of which the leading ones are illustrated in Fig. 2. The relevance of such contributions depends on the Higgs mass and on the centre-of-mass (c.m.) energy: the dominant decay mode is $H \rightarrow b \bar{b}$ for low Higgs masses $\left(m_{H} \leqslant 130-140 \mathrm{GeV}\right)$ and $H \rightarrow W(V=W, Z)$ for 
high Higgs masses. The predictions can thus be expected to depend on the Higgs mass.

As is well known, the behaviour of the cross section near the threshold for $t \bar{t}$ production is characterized by strong interaction effects that give a sizeable modification with respect to the purely electroweak prediction. Such effects are treated in the literature $[11,12]$, and are not included in the calculations presented in this paper. ${ }^{1}$ Results at energies around the threshold are shown, so as to give a thorough analysis of the electroweak contribution. Some of the QCD backgrounds to the signatures considered in the present study have been evaluated in Ref. [13], and their topology has been studied by means of event-shape variables. One of the objectives of the present work is to characterize the topology of the complete electroweak contributions in order to help finding appropriate selection criteria to reduce as far as possible the QCD backgrounds studied in Ref. [13]. The analysis performed in the present work, together with the other studies in the literature so far, should give a complete picture of electroweak contributions to $6 f$ processes relevant to top-quark physics at NLC.

The paper is organized as follows: in Section 2 the computing procedure is briefly described; in Section 3 the numerical results, including integrated cross sections and various distributions, are presented and discussed; Section 4 is devoted to our conclusions.

\section{Calculation}

The numerical results have been obtained by means of a procedure analogous to the one adopted in Ref. [8], where the interested reader can find some technical details that will be omitted here. The computer program already used in Ref. [8], which is based on ALPHA [14] for the matrix element calculation and on an evolution of HIGGSPV/WWGENPV [15,16] for the Monte Carlo integration and event generation, has been adapted, in the multichannel importance sampling, to include some new diagram topologies, such as those in Figs. 1 and 2.

The cross section is calculated according to the formula

$$
\sigma=\int d z_{1} d z_{2} D_{\mathrm{BS}}\left(z_{1}, z_{2} ; s\right) \int d x_{1} d x_{2} D\left(x_{1}, s\right) D\left(x_{2}, s\right) d[\mathrm{PS}] \frac{d \hat{\boldsymbol{\sigma}}}{d[\mathrm{PS}]},
$$

where initial-state radiation (ISR) [17] and beamstrahlung (BS) [18] are included by means of the structure functions $D(x, s)$ and $D_{\mathrm{BS}}(x, s)$, respectively; $d \hat{\sigma} / d[\mathrm{PS}]$ is the differential cross section at the partonic level, and $d$ [PS] is the six-body phase-space measure. The program may be used to generate unweighted events as well.

The input parameters are $G_{\mu}, M_{W}, M_{Z}$, the top-quark mass $m_{t}=175 \mathrm{GeV}$, and the $b$-quark mass $m_{b}=4.3 \mathrm{GeV}$; all the other fermions are treated as massless. The widths of the $W$ and $Z^{0}$ bosons and of the top-quark and all the couplings are calculated at tree level. The Higgs-boson width includes the $h \rightarrow \mu \mu, \tau \tau, c c, b b$, the $h \rightarrow g g$ [19]

\footnotetext{
${ }^{1}$ Theoretical calculations of radiative corrections to $t \bar{t}$ production are also present in the literature, as recently reviewed in Ref. [12].
} 
and the two-vector-boson [20] channels. The CKM matrix used is exactly diagonal. The propagators of unstable particles have denominators of the form $p^{2}-M^{2}+i \Gamma M$ with fixed widths. The validity of this choice for minimizing possible gauge violations has been discussed in Ref. [8], where the final states $q \bar{q} l^{+} l^{-} \nu \bar{\nu}$ were considered. In that paper, for the $S U(2)$ invariance, the fudge-factor method has been used to check the numerical results: apart from the well-known problems of the fudge scheme, i.e. the mistreatment of non-resonant diagrams close to the resonances, no deviation has been found in the total cross section up to the numerical accuracy considered. In order to check $U(1)$ invariance, the matrix element has been calculated with different forms of the photon propagator obtained by varying the gauge parameter; the results were found to be stable up to numerical precision. The same analysis carries on to the present study and gauge-violation effects are estimated to be numerically negligible.

The colour algebra, not implemented in the version of ALPHA that has been employed here, has been performed by summing the different processes with proper weights. As an example of this, the process $e^{+} e^{-} \rightarrow b \bar{b} u \bar{d} \bar{u} d$ may be considered: the colour amplitude, in the case of purely electroweak contributions, can be written in the form

$$
A=\left(a_{1} \delta_{i_{1} i_{2}} \delta_{i_{3} i_{4}}+a_{2} \delta_{i_{1} i_{3}} \delta_{i_{2} i_{4}}\right) \delta_{j k}
$$

where the colour indices $i_{1}, i_{2}, i_{3}, i_{4}, j$ and $k$ refer to the $u, \bar{d}, \bar{u}, d, b$ and $\bar{b}$ quarks respectively. The squared modulus summed over colours is then

$$
\sum_{\text {col }}|A|^{2}=N_{c}^{3}\left|a_{1}\right|^{2}+N_{c}^{2}\left(a_{1} a_{2}^{*}+a_{1}^{*} a_{2}\right)+N_{c}^{3}\left|a_{2}\right|^{2} .
$$

The amplitude given by ALPHA is instead

$$
\mathcal{A}=a_{1}+a_{2} .
$$

Thus one cannot use an overall factor to obtain Eq. (3) from Eq. (4). In order to disentangle the various terms in Eq. (3), it is useful to notice that, with the quark masses adopted here and with a diagonal CKM matrix, the first term in the right-hand side of Eq. (2) is equal to the amplitude $A^{\prime}$ of the process $e^{+} e^{-} \rightarrow b \bar{b} u \bar{d} \bar{c} s$, and the second term is equal to the amplitude $A^{\prime \prime}$ of the process $e^{+} e^{-} \rightarrow b \bar{b} u \bar{s} \bar{u} s$. Similarly, the two colourless amplitudes $\mathcal{A}^{\prime}$ and $\mathcal{A}^{\prime \prime}$ of these processes are equal to the first and to the second term, respectively, in the right-hand side of Eq. (4). Thus the following relation is valid:

$$
\sum_{\text {col }}\left(|A|^{2}+\left|A^{\prime}\right|^{2}+\left|A^{\prime \prime}\right|^{2}\right)=N_{c}^{2}\left(|\mathcal{A}|^{2}+\left(2 N_{c}-1\right)\left(\left|\mathcal{A}^{\prime}\right|^{2}+\left|\mathcal{A}^{\prime \prime}\right|^{2}\right)\right)
$$

Other situations are treated in a similar way, and the correct colour weights are thus obtained in the sum over the whole class of processes considered. 


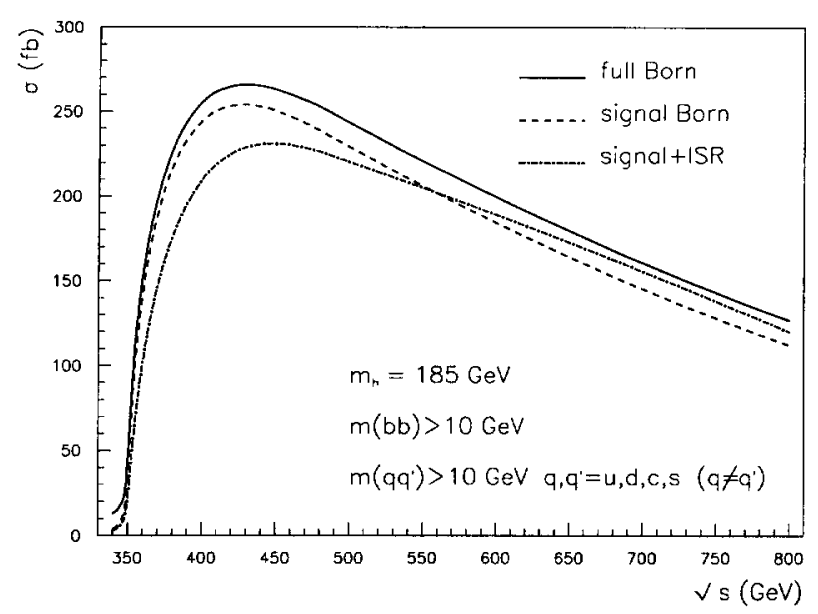

Fig. 3. Full six-quark electroweak cross section (solid line) and $t \bar{f}$ signal (dashed line) in the Born approximation, and $t \bar{t}$ signal with initial-state radiation (dash-dotted line), as a function of the c.m. energy.

\section{Numerical results and discussion}

In this section the numerical results, including both integrated cross sections and distributions, are shown. In all the calculations the invariant masses of the $b \bar{b}$ pair and of all the pairs of quarks other than $b$ and $\bar{b}$ are required to be greater than $10 \mathrm{GeV}$. The results presented below are obtained, unless otherwise stated, by summing over all the processes listed in Table 1.

\subsection{Integrated cross sections}

As a first step, the total cross section, resulting from all the tree-level diagrams for the processes in Table 1, has been calculated in the Born approximation at energies from 340 to $800 \mathrm{GeV}$. Two values of Higgs mass have been considered, $m_{H}=100,185 \mathrm{GeV}$, so as to study the dependence of the results on $m_{H}$ in the intermediate range. The numerical errors are always below $1 \%$ and in particular above the $t \bar{t}$ threshold they are kept at $0.2-0.3 \%$ level.

In Fig. 3 the full cross section for $m_{H}=185 \mathrm{GeV}$ is compared with the signal, defined as the contribution of the two diagrams of $t \bar{t}$ production of Fig. 1, summed over the four processes to which they contribute (see the first two columns of Table 1); the signal is shown both in the Born approximation and with ISR switched on.

The difference between the full and the signal curve is dominated by Higgs-strahlung contributions (diagrams (a) and (b) of Fig. 2) at low energy, while other backgrounds are important at high energy, coming from all the processes in Table 1 and, for a little amount, from the interference of the signal diagrams with the other contributions in the charged-current and mixed processes. The electroweak background effects, which are in the range 5-10\% above the threshold, amount to around $30 \%$ at threshold; they are much greater below the threshold, where the signal is sup- 


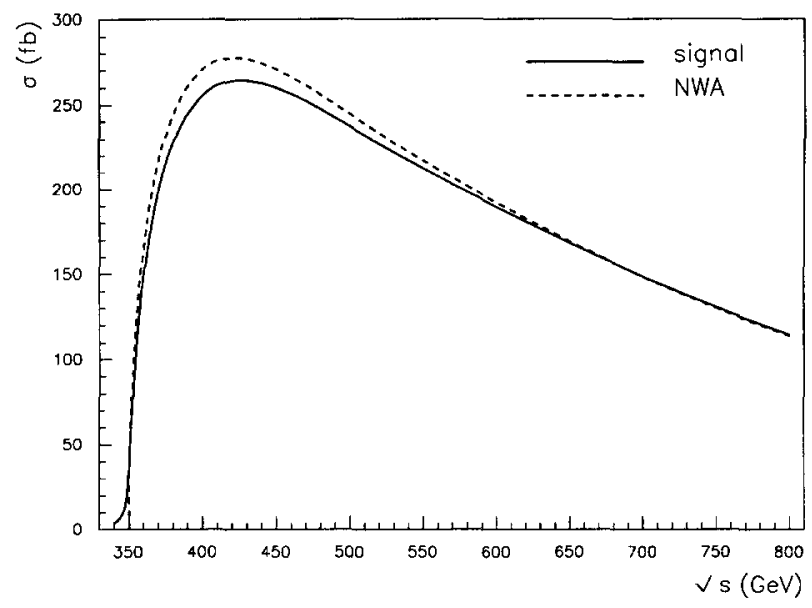

Fig. 4. Signal cross section (solid line) without cuts compared with the narrow-width approximation (dashed line), as a function of the c.m. energy.

pressed with respect to the background (the ratio background/signal is of 2.5 at $340 \mathrm{GeV}$ ).

The radiative effects strongly suppress the cross section in the low-energy region, where it grows rapidly, as they reduce the effective c.m. energy; with increasing energy, the curve with ISR comes to cross the one in the Born approximation, as a consequence of the onset of the opposite behaviour of the Born term, which, above the threshold, starts decreasing. It can be observed that at $500 \mathrm{GeV}$ the enhancement due to the background is of the same order as the lowering given by the ISR.

In Fig. 4 the signal cross section without kinematical cuts is plotted together with the cross section in the narrow-width approximation (NWA). The latter is calculated as the product of the cross section for $e^{+} e^{-} \rightarrow t \bar{t}$, and of the branching ratios of the decays $W \rightarrow q \bar{q}^{\prime}$, assuming the branching ratio of $t \rightarrow W b$ to be exactly unity. The difference between the two calculations is about $15 \%$ in the region near the threshold, and it decreases, as expected, with increasing c.m. energy: at $500 \mathrm{GeV}$ it is $3 \%$, while at $800 \mathrm{GeV}$ it is less than $1 \%$. These results give a measure of the off-shellness effects connected with the top-quark and $W$-boson widths.

The cross sections for the two values of the Higgs mass, $m_{H}=100$ and $185 \mathrm{GeV}$, have been found to differ by less than $1 \%$ at energies above the threshold region, while at lower energies, differences of up to $20-30 \%$ occur. This is due to the fact that the signal at low energy is not large enough to hide the Higgs-mass effects. Moreover, such effects decrease with increasing energy. In order to make a detailed study of the dependence on the Higgs mass at low energy, the cross section at the threshold for $t \bar{t}$ production, $\sqrt{s}=350 \mathrm{GeV}$, has been calculated for various Higgs masses in the range from $100 \mathrm{GeV}$ to $185 \mathrm{GeV}$. The results are shown in Fig. 5, where the cross section is plotted as a function of the Higgs mass. Variations of the order of $10 \%$ can be seen in this plot, which shows the importance of complete calculations to keep under control the background effects and uncertainties that come from not knowing the Higgs mass. 


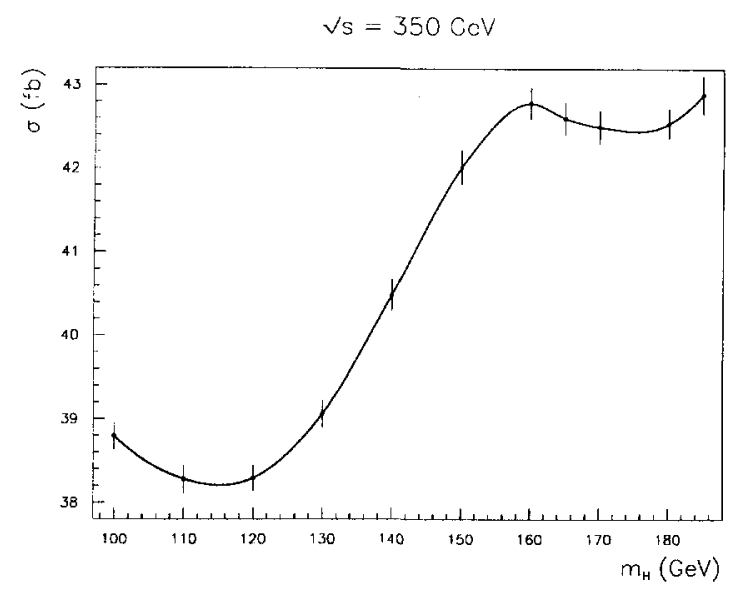

Fig. 5. Total cross section as a function of the Higgs mass at the threshold for $t \bar{t}$ production.

\subsection{Distributions}

Two samples of events have been generated at a c.m. energy of $500 \mathrm{GeV}$ and with a Higgs mass of $185 \mathrm{GeV}$. One sample is in the Born approximation, while the other includes ISR and BS. The numbers of events, of the order of $10^{5}$, have been determined by assuming a luminosity of $500 \mathrm{fb}^{-1}$, which is the integrated value expected in one year of run.

In the definition of observable distributions for the class of processes considered here, we must take into account the fact that quark flavours other than $b$ cannot be identified. As a consequence, two kinds of distributions, labelled "exact" and "reconstructed", are considered in the following: the "exact" distributions are calculated by identifying all the quarks; the "reconstructed" distributions are calculated by means of the following algorithm. The momenta $q_{1}, \ldots, q_{4}$ of the four quarks other than $b$ and $\bar{b}$ are first considered and, for every pair $\left(q_{i}, q_{j}\right)$, the invariant mass $m_{i j}=\sqrt{\left(q_{i}+q_{j}\right)^{2}}$ is calculated; then the two $W$ particles, $W_{1}$ and $W_{2}$, are reconstructed as the pairs $\left(q_{i}, q_{j}\right)$ and $\left(q_{k}, q_{l}\right)$ such that the quantity $\left|m_{i j}-M_{W}\right|+\left|m_{k l}-M_{W}\right|$ is minimized; the top-quark is then determined by taking the combination $\left(b, W_{i}\right),\left(\bar{b}, W_{j}\right)$, which minimizes the quantity $\left|m_{b W_{i}}-\tilde{m}_{t}\right|+\left|m_{\tilde{b} W_{j}}-\tilde{m}_{t}\right|$, where $\tilde{m}_{t}=175 \mathrm{GeV}$ is the nominal top mass.

The invariant mass of the top-quark is studied in Fig. 6. In the plot (a) a comparison is made between the exact (dashed line) and the reconstructed (solid line) distribution in the Born approximation and a good agreement can be observed. In order to further check the reconstruction procedure, in particular the dependence on the adopted value of $\tilde{m}_{t}$, some tests have been made by taking values in the range $170 \mathrm{GeV}<\tilde{m}_{t}<180$ $\mathrm{GeV}$ and fitting the resulting histograms with Breit-Wigner distributions. The values of the physical top mass obtained in the various cases are identical, within the statistical errors.

The radiative effects are shown in the plot (b) of Fig. 6, for the reconstructed distribution. They do not apparently give a substantial modification. In the plot (c) the 


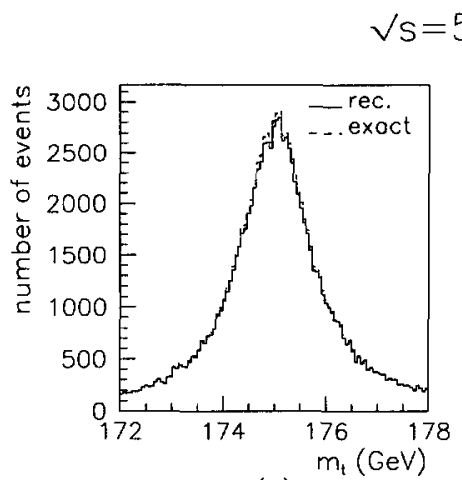

(a)

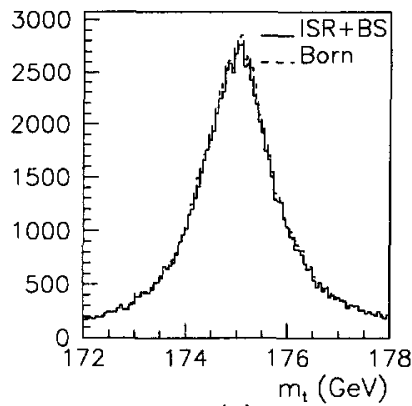

(b)

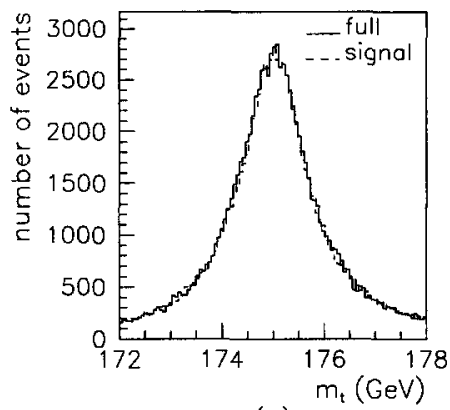

(c)

Fig. 6. Invariant mass of the top-quark. (a) Exact (solid line) and reconstructed (dashed line) distribution; (b) distribution in the Born approximation (dashed line) and with initial-state radiation and beamstrahlung (solid line); (c) full calculation (solid line) and signal (dashed line).

role of background diagrams is studied, by comparing the result of the full calculation with the signal alone. The background does not introduce any observable distortion. More quantitative results have been obtained by means of fits to the histograms with Breit-Wigner distributions. All the histograms in Fig. 6 give the same value of $m_{t}$, so that it can be safely concluded that electroweak backgrounds, as well as ISR and BS do not give any bias in the determination of the physical mass via the direct reconstruction method on the scale of precision of $100 \mathrm{MeV}$.

The angular distribution of the top-quark with respect to the beam axis is a good indicator of the spin nature and of the couplings of the top-quark. This variable is illustrated in Fig. 7. As in the case of the invariant mass, the exact and reconstructed distributions have been checked to be in very good agreement. It should be observed that radiative and background effects are of the same magnitude (in particular the former are dominated by the ISR). The shapes of the histograms are in good qualitative agreement with the angular distribution predicted by the lowest-order analytic calculation for the process of real $t \bar{t}$ production.

The most effective way to obtain a separation between the $t \tilde{t}$ signal and the QCD backgrounds, as already pointed out by some authors $[13,21]$, is to analyse event-shape variables, such as thrust [22], sphericity [23], spherocity [24], $C$ and $D$ parame- 


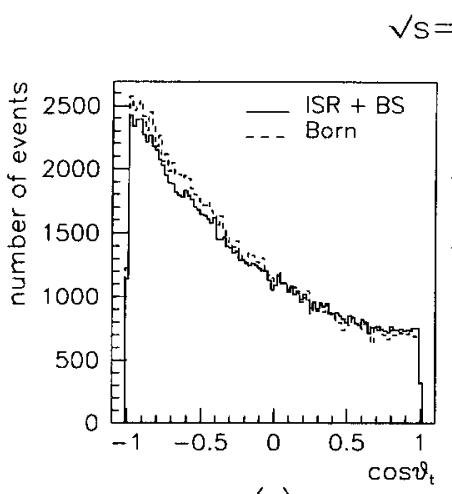

(a)

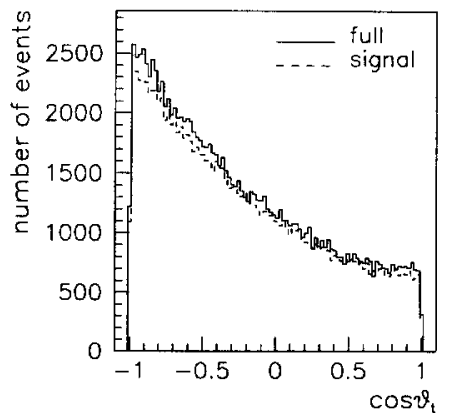

(b)

Fig. 7. Reconstructed angular distribution of the top-quark with respect to the $e^{+}$beam axis. (a) Results in the Born approximation (dashed histogram) and with initial-state radiation and beamstrahlung (solid histogram). (b) Full calculation (solid line) and signal contribution (dashed line).

ters [25], etc. A comparison between pure QCD $\left(O\left(\alpha_{e m}^{2} \alpha_{s}^{4}\right)\right)$ six-jet events and the $t \bar{t}$ signal has been performed in Ref. [13] for the thrust and sphericity distributions, and other shape variables have been studied in the same article for the QCD contributions only. In the present work several such variables have been analysed for the electroweak contributions, and the effects of the electroweak backgrounds and of ISR and BS have been studied. The thrust and $C$ parameter distributions for the process under consideration are shown in Fig. 8. In the upper row the radiative effects are displayed, while in the plots of the lower row the signal is compared with the full result. In the radiative case, the distributions are calculated after going to the c.m. frame. Remarkable effects due to ISR and BS can be seen in these plots and in particular in the thrust distribution, where the peak is strongly reduced with respect to the Born approximation and the events are shifted towards the lower values of $T$, which correspond to spherical events. It is interesting to observe that this phenomenon is of help for the selection of the signal with respect to QCD backgrounds. From the plots in the second row, it can be seen that the presence of the electroweak backgrounds, although visible, is almost negligible for both observables.

The remarkable change of the thrust distribution after inclusion of radiation can be better understood by observing the dependence of this distribution on the c.m. energy, which is analysed in Fig 9, where four samples of 10000 events each, at the energies of $360,500,800$ and $1500 \mathrm{GeV}$, are studied. The peak structure that is present at $500 \mathrm{GeV}$ is completely lost at $360 \mathrm{GeV}$, and this explains the lowering of the peak at $500 \mathrm{GeV}$ in the presence of ISR and BS, as this reduces the available c.m. energy. At 800 and $1500 \mathrm{GeV}$ the peak is shifted towards the collinear region $T \sim 1$, as a consequence of the Lorentz boost of the $t$ and $\bar{t}$ quarks.

As a conclusion, we can say that, at $500 \mathrm{GeV}$, in view of the results of the pure QCD processes, studied in Ref. [13], the thrust variable is the most effective in discriminating pure QCD backgrounds, also in the presence of electroweak backgrounds and of ISR and BS. At higher energies this separation appears to be more and more problematic. 


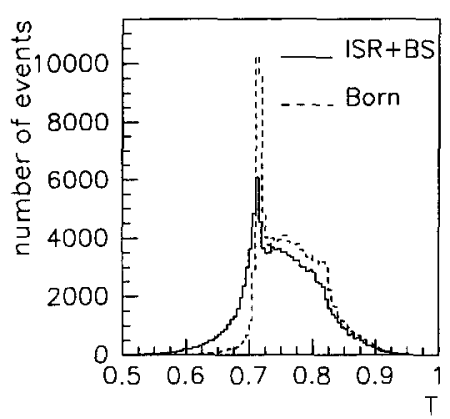

(a)

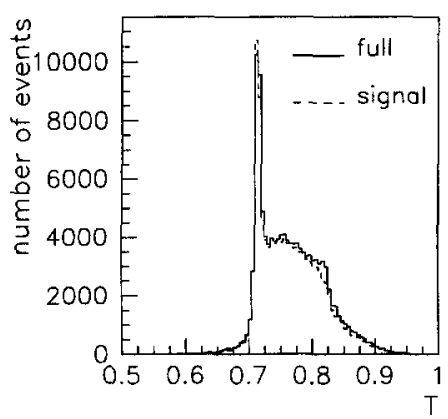

(c)

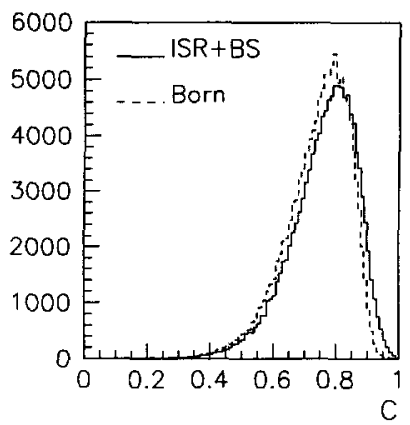

(b)

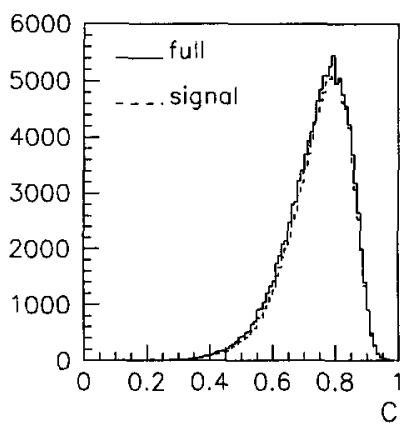

(d)

Fig. 8. Event-shape variables. (a) Thrust distribution in the Born approximation (dashed histogram) and with initial-state radiation and beamstrahlung (solid histogram); (b) $C$ parameter distribution, as in (a); (c) thrust distribution in the Born approximation from the full calculation (solid histogram) and from the signal contributions alone (dashed histogram); (d) $C$ parameter distribution, as in (c).

On the other hand, the backgrounds of $O\left(\alpha_{e m}^{4} \alpha_{s}^{2}\right)$, given by $2 \rightarrow 4$ processes with subsequent gluon emission from a quark line, should be considered (a study of contributions of this class for semi leptonic signatures is made in Ref. [10], but without an analysis of event-shape variables). A rough estimate of the leading contributions of this kind could be obtained by considering a four-fermion process of the form $e^{+} e^{-} \rightarrow W^{+} W^{-} \rightarrow 4$ jets, similar to what is done in Ref. [21]. A test made by means of the four-fermion program WWGENPV has confirmed the results of Ref. [21] for the thrust and has led to similar conclusions for the $C$ parameter: such processes appear to be well separated from the top-quark signal and thus appear to be less dangerous than the pure QCD backgrounds.

\section{Conclusions}

The production of $t \bar{t}$ pairs has been studied in processes with six quarks in the final state, at the energies of the NLC. The signatures considered contain one $b \bar{b}$ pair, and receive contributions from both charged and neutral currents. The top-quark signal is present only in the charged-current terms. The purely electroweak contributions have been considered and complete tree-level calculations have been performed. 

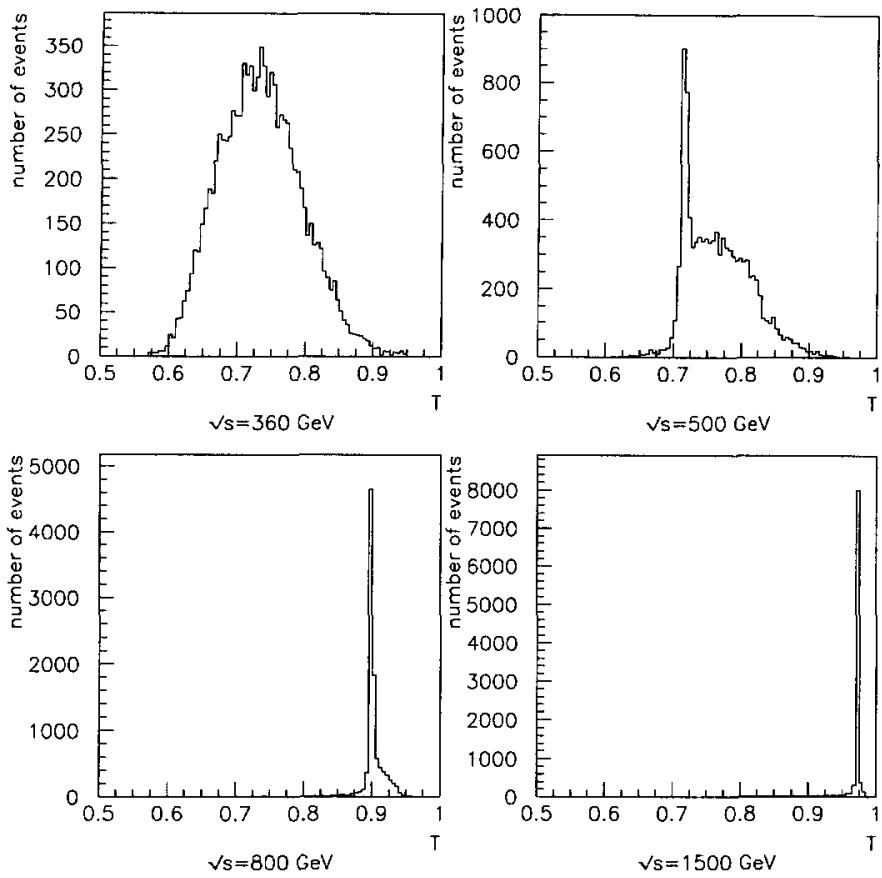

Fig. 9. Thrust distribution in the Born approximation at $360,500,800$ and $1500 \mathrm{GeV}$.

The cross section has been calculated by means of a computer program already used for other phenomenological studies on $6 f$ processes and adapted here to sample the new diagram topologies. The importance of the electroweak backgrounds and of the offshellness effects has been examined. Above the threshold for $t \bar{t}$ production, the former are of the order of several per cent and the latter are at the per cent level. Near the threshold, both effects are sizeable and, in particular, a study of the dependence of the cross section on the Higgs mass at threshold shows that variations of the order of $10 \%$ occur for Higgs masses between $100 \mathrm{GeV}$ and $185 \mathrm{GeV}$. A complete calculation is needed to keep such effects under control and to have a $1 \%$ accuracy.

Some distributions have been studied in a realistic approach, by using a reconstruction algorithm for the top-quark that takes into account the impossibility of identifying quark flavours other than $b$. The invariant mass of the top-quark has thus been studied and the presence of electroweak background contributions as well as the initial-state radiative effects have been found not to affect the determination of the mass on the scale of experimental precision expected at NLC.

The angular distribution of the top-quark with respect to the beam axis, which is directly related, in the case of real production, to the quantum numbers of the top-quark, has been calculated and shown to be in qualitative agreement with the expectation suggested by the real production case.

Finally, some event-shape variables have been studied. At a c.m. energy of $500 \mathrm{GeV}$ the thrust distribution turns out to be the most interesting for the aim of discriminating 
the leading QCD backgrounds, as suggested by other authors who discussed the topquark signal alone. The effects of electroweak backgrounds and of ISR and BS have been shown here not to alter these conclusions. At higher energies, the Lorentz boost gives to the event a more collinear shape, so that the separation of QCD backgrounds could become more difficult.

The study presented in this paper has been performed by means of a computing program that can equally well deal with semi leptonic signatures and can be switched in a straightforward manner to treat polarized scattering. Moreover, by employing the new version of ALPHA [26], which embodies also the QCD Lagrangian, complete strong and electroweak results could be obtained.

\section{Acknowledgements}

F. Gangemi thanks the INFN, Sezione di Pavia, for the use of computing facilities. The work of M. Moretti is funded by a Marie Curie fellowship (TMR-ERBFMBICT 971934).

\section{References}

[1] E. Accomando et al., Phys. Rep. 299 (1998) 1;

F. Boudjema, Pramana 51 (1998) 249.

[2] G. Jarlskog and D. Rein (ed.), Proc. of the Large Hadron Collider Workshop, Aachen, 1990; ATLAS Technical Proposal, CERN/LHC/94-43 LHCC/P2 (December 1994); CMS Technical Proposal, CERN/LHC/94-43 LHCC/P1 (December 1994).

[3] P. Bhat, H. Prosper and S. Snyder, Int. J. Mod. Phys. A 13 (1998) 5113.

[4] F. Yuasa, Y. Kurihara and S. Kawabata, Phys. Lett. B 414 (1997) 178.

[5] E. Accomando, A. Ballestrero and M. Pizzio, Nucl. Phys. B 512 (1998) 19; in R. Settles (ed.) " $e^{+} e^{-}$ Linear Colliders: Physics and Detector Studies, Part E", DESY 97-123E, p. 31; Nucl. Phys. B 547 (1999) 81;

A. Ballestrero, Acta Phys. Pol. B 29 (1998) 2811.

[6] G. Montagna, M. Moretti, O. Nicrosini and F. Piccinini, Eur. Phys. J. C 2 (1998) 483.

[7] F. Gangemi, G. Montagna, M. Moretti, O. Nicrosini and F. Piccinini in R. Settles (ed.) " $e^{+} e^{-}$Linear Colliders: Physics and Detector Studies, Part E", DESY 97-123E, p. 393.

[8] F. Gangemi, G. Montagna, M. Moretti, O. Nicrosini and F. Piccinini, Eur. Phys. J. C9 (1999) 31.

[9] T. Ohl, talk given at the Workshop on Physics and Detectors for Future $e^{+} e^{-}$Linear Colliders, 26-29 September 1998, Keystone (CO).

[10] S. Moretti, Eur. Phys. J. C9 (1999) 229.

[11] V.S. Fadin and V.A. Khoze, Sov. J. Nucl. Phys. 48 (1988) 309; M.J. Strasseler and M. Peskin, Phys. Rev. D 43 (1991) 1500.

[12] T. Teubner, Acta Phys. Pol. B 30 (1999) 1941, and references therein.

[13] S. Moretti, Phys. Lett. B 420 (1998) 367; Nucl. Phys. B 544 (1999) 289.

[14] F. Caravaglios and M. Moretti, Phys. Lett. B 358 (1995) 332.

[15] Program HIGGSPV, by G. Montagna, O. Nicrosini and F. Piccinini; write up in $[28,29]$.

[16] Program WWGENPV, by G. Montagna, O. Nicrosini and F. Piccinini; write up in [28,29]; See also: G. Montagna, O. Nicrosini and F. Piccinini, Comput. Phys. Commun. 90 (1995) 141; D.G. Charlton, G. Montagna, O. Nicrosini and F. Piccinini, Comput. Phys. Commun. 99 (1997) 355.

[17] E.A. Kuraev and V.S. Fadin, Sov. J. Nucl. Phys. 41 (1985) 466; G. Altarelli and G. Martinelli, in: Physics at LEP, ed. J. Ellis and R. Peccei, CERN Report 86-02 
(Geneva, 1986), Vol. 1, p. 47;

O. Nicrosini and L. Trentadue, Phys. Lett. B 196 (1987) 551; Z. Phys. C 39 (1988) 479;

F.A. Berends, G. Burgers and W.L. van Neerven, Nucl. Phys. B 297 (1988) 429.

[18] T. Ohl, Comput. Phys. Commun. 101 (1997) 269.

[19] M. Carena, P.M. Zerwas (convenors), "Higgs Physics", in [27], Vol. 1, p. 351.

[20] B. Kniehl, Phys. Lett. B 244 (1990) 537.

[21] P. Igo-Kimenes (convener) and J.H. Kühn (advisor), in: Proc. of the Workshop on " $e^{+} e^{-}$Collisions at $500 \mathrm{GeV}$. The Physics Potential", Munich, Annecy, Hamburg, ed. P.M. Zerwas, DESY 92-123, 1992, part A, p. 327

[22] S. Brandt, Ch. Peyrou, R. Sosnowski and A. Wroblewski, Phys. Lett. 12 (1964) 57; E. Farhi, Phys. Rev. Lett. 39 (1977) 1587.

[23] J.D. Bjorken and S. Brodsky, Phys. Rev. D 1 (1970) 1416.

[24] H. Georgi and M. Machacek, Phys. Rev. Lett. 39 (1977) 1237.

[25] G. Parisi, Phys. Lett. B 74 (1978) 65;

J.F. Donoghue, F.E. Low and S.Y. Pi, Phys. Rev. D 20 (1979) 2759;

R.K. Ellis, D.A. Ross and A.E. Terrano, Nucl. Phys. B 178 (1981) 421.

[26] F. Caravaglios, M.L. Mangano, M. Moretti and R. Pittau, Nucl. Phys. B 539 (1999) 215.

[27] G. Altarelli, T. Sjöstrand and F. Zwirner, (ed.), Physics at LEP2, CERN Report 96-01 (Geneva, 1996), Vols. 1 and 2 .

[28] M.L. Mangano, G. Ridolfi (convenors), "Event Generators for Discovery Physics", in [27], Vol. 2, p. 299.

[29] D. Bardin, R. Kleiss (convenors), "Event Generators for WW Physics", in [27], Vol. 2, p. 3. 